\title{
Travelling Waves of an n-Species Food Chain Model with Spatial Diffusion and Time Delays
}

\author{
Fei Hu, Yuyin Xu, Z. Wang, and Wei Ding \\ Department of Mathematics, Shanghai Normal University, Shanghai 200234, China \\ Correspondence should be addressed to Wei Ding; dingwei@shnu.edu.cn
}

Received 21 February 2014; Accepted 28 March 2014; Published 20 May 2014

Academic Editor: Yonghui Xia

Copyright ( 92014 Fei Hu et al. This is an open access article distributed under the Creative Commons Attribution License, which permits unrestricted use, distribution, and reproduction in any medium, provided the original work is properly cited.

We investigate an $n$-species food chain model with spatial diffusion and time delays. By using Schauder's fixed point theorem, we obtain the result about the existence of the travelling wave solutions of the food chain model with reaction term satisfying the partial quasimonotonicity conditions.

\section{Introduction}

In the past few decades, the dynamic relationship between predators and preys has been investigated intensively due to its universal existence and importance in both ecology and mathematical ecology [1-3].

Recently, traveling waves for reaction-diffusion systems (see, e.g., [4-7]) have received considerable attention since they determine the long-term behavior of other solutions in many situations. By now, many powerful methods have been used to study the travelling wave solutions for reactiondiffusion systems, like phase plane techniques in [8], degree theory methods $[9,10]$, the shooting methods [11], the monotone iteration [1], and so on [12,13].

Although the existence of travelling wave solutions to reaction-diffusion systems without delay has been widely studied [14-16], delayed reaction-diffusion systems which are more realistic in population dynamic and biological models are much more complicated than ordinary systems. Recently, a number of researchers have studied the existence of travelling wave solutions in delayed reactiondiffusion systems. In [17, 18], Wu and Zou considered delayed reaction-diffusion systems with reaction terms satisfying the so-called quasimonotonicity or exponential quasimonotonicity conditions. In [19], Huang and Zou employed the upper-lower solution technique and the monotone to study the existence of travelling wave solutions for a class of diffusion cooperative Lotka-Volterra systems with delays. In [20], Ma used Schauder's fixed point theorem to study the existence of travelling wave solutions to reaction-diffusion systems with quasimonotonicity reaction terms. In 2010, Gan et al. [21] investigated the existence of travelling wave solutions to the following three-species food chain models:

$$
\begin{gathered}
\frac{\partial u}{\partial t}=D_{1} \frac{\partial^{2} u}{\partial x^{2}}+u(t, x)\left(r_{1}-a_{11} u(t, x)-a_{12} v(t, x)\right), \\
\frac{\partial v}{\partial t}=D_{2} \frac{\partial^{2} v}{\partial x^{2}}+v(t, x)\left(-r_{2}+a_{21} v(t-\tau, x)\right. \\
\left.-a_{22} v(t, x)-a_{23} w(t, x)\right), \\
\frac{\partial w}{\partial t}=D_{3} \frac{\partial^{2} w}{\partial x^{2}}+w(t, x)\left(-r_{3}+a_{32} v(t-\tau, x)-a_{33} w(t, x)\right) .
\end{gathered}
$$

They obtain the existence of traveling waves by using Schauder's fixed point theorem with the reaction term satisfying the partial quasimonotonicity conditions instead of the quasimonotonicity conditions. 
Motivated by the above papers, in this paper, we investigate an $n$-species food chain model with spatial diffusion and time delays:

$$
\begin{aligned}
\frac{\partial u_{i}(t, x)}{\partial t}= & D_{i} \frac{\partial^{2} u_{i}(t, x)}{\partial x^{2}}-a_{i} u_{i}(t, x)+\sum_{j=1}^{n} w_{i j} f_{j}\left(u_{j}(t, x)\right) \\
& +\sum_{j=1}^{n} h_{i j} g_{j}\left(u_{j}\left(t-\tau_{j}(t), x\right)\right),
\end{aligned}
$$

where $D_{i}, a_{i}, w_{i j}$, and $h_{i j}$ are positive constants and $i=$ $1,2, \ldots, n$.

The main propose of this paper is to obtain the sufficient condition for the existence of the travelling wave solutions of system (2) by employing Schauder's fixed point theorem. This paper is organized as follows. In Section 2, some definition and lemmas are given. And the main results of the paper are established in the last section.

\section{Preliminary}

On substituting $u_{i}(t, x)=\phi_{i}(x+c t)$ and denoting the traveling wave coordinate $x+c t$ still by $t$, we derive from (2) that

$$
\begin{aligned}
D_{i} \phi_{i}^{\prime \prime}(t)-c \phi_{i}^{\prime}(t)-a_{i} \phi_{i}(t)+\sum_{j=1}^{n} w_{i j} f_{j}\left(\phi_{j}(t)\right) \\
+\sum_{j=1}^{n} h_{i j} g_{j}\left(\phi_{j}\left(t-\tau_{j}(t)\right)\right)=0 .
\end{aligned}
$$

If for some $c>0$, system (2) has a solution defined on $R^{n}$ satisfying

$$
\lim _{t \rightarrow-\infty} \phi_{i}(t)=\phi_{i}^{-}, \quad \lim _{t \rightarrow \infty} \phi_{i}(t)=\phi_{i}^{+},
$$

where $\left(\phi_{1}^{-}, \phi_{2}^{-}, \ldots, \phi_{n}^{-}\right)$and $\left(\phi_{1}^{+}, \phi_{2}^{+}, \ldots, \phi_{n}^{+}\right)$are steady states of (2), then $u_{i}(t, x)=\phi_{i}(x+c t)$ is called a traveling wave solution of (2) with speed $c$. Without loss of generality, we assume that $\left(\phi_{1}^{-}, \phi_{2}^{-}, \ldots, \phi_{n}^{-}\right)=(0,0, \ldots, 0)$ and $\left(\phi_{1}^{+}, \phi_{2}^{+}, \ldots, \phi_{n}^{+}\right)=$ $\left(k_{1}, k_{2}, \ldots, k_{n}\right)$.

Rewrite model (3) as

$$
D_{i} \phi_{i}^{\prime \prime}(t)-c \phi_{i}^{\prime}(t)-a_{i} \phi_{i}(t)+H_{i}\left(\phi_{1}, \phi_{2}, \ldots, \phi_{n}\right)(t)=0,
$$

where

$$
\begin{aligned}
& H_{i}\left(\phi_{1}, \phi_{2}, \ldots, \phi_{n}\right)(t) \\
& \quad=\sum_{j=1}^{n} w_{i j} f_{j}\left(\phi_{j}(t)\right)+\sum_{j=1}^{n} h_{i j} g_{j}\left(\phi_{j}\left(t-\tau_{j}(t)\right)\right) .
\end{aligned}
$$

In the following parts, we assume that the nonlinear reaction terms $H_{i}\left(\phi_{1}, \phi_{2}, \ldots, \phi_{n}\right)(t)(i=1,2, \ldots, n)$ satisfy the partial quasimonotonicity conditions (PQM):
(1) $H_{n}$ is nondecreasing. That is, for $0 \leq \underline{\phi}_{j} \leq \phi_{j} \leq \bar{\phi}_{j} \leq$ $k_{j}(j=1,2, \ldots, n)$,

$$
\begin{aligned}
& H_{n}\left(\underline{\phi}_{1}, \underline{\phi}_{2}, \ldots, \underline{\phi}_{n}\right) \\
& \quad \leq H_{n}\left(\phi_{1}, \phi_{2}, \ldots, \phi_{n}\right) \leq H_{n}\left(\bar{\phi}_{1}, \bar{\phi}_{2}, \ldots, \bar{\phi}_{n}\right) .
\end{aligned}
$$

(2) For $t \in R$, and $0 \leq \underline{\phi}_{j} \leq \phi_{j} \leq \bar{\phi}_{j} \leq k_{j}(j=1,2, \ldots, n)$,

$$
\begin{array}{r}
H_{i}\left(\underline{\phi}_{1}, \underline{\phi}_{2}, \ldots, \underline{\phi}_{i}, \bar{\phi}_{i+1}, \underline{\phi}_{i+2}, \ldots, \underline{\phi}_{n}\right)(t) \\
\leq H_{i}\left(\phi_{1}, \phi_{2}, \ldots, \phi_{n}\right)(t) \\
\leq H_{i}\left(\bar{\phi}_{1}, \bar{\phi}_{2}, \ldots, \bar{\phi}_{i}, \underline{\phi}_{i+1}, \bar{\phi}_{i+2}, \ldots, \bar{\phi}_{n}\right)(t), \\
i=1,2, \ldots, n-1 .
\end{array}
$$

Let $C_{k}\left(R, R^{n}\right)=\left\{\left(\phi_{1}, \phi_{2}, \ldots, \phi_{n}\right) \in C\left(R, R^{n}\right): 0 \leq \phi_{i} \leq\right.$ $\left.k_{i}, i=1,2, \ldots, n\right\}$, and for $\left(\phi_{1}, \phi_{2}, \ldots, \phi_{n}\right) \in C_{k}\left(R, R^{n}\right)$, define $F=\left(F_{1}, F_{2}, \ldots, F_{n}\right): C_{k}\left(R, R^{n}\right) \rightarrow C_{k}\left(R, R^{n}\right)$ by

$$
\begin{aligned}
& F_{i}\left(\phi_{1}, \phi_{2}, \ldots, \phi_{n}\right)(t) \\
& =\frac{1}{D_{i}\left(\lambda_{i 2}-\lambda_{i 1}\right)} \\
& \quad \times\left[\int_{-\infty}^{t} e^{\lambda_{i 1}(t-s)} H_{i}\left(\phi_{1}, \phi_{2}, \ldots, \phi_{n}\right)(s) d s\right. \\
& \left.\quad \quad+\int_{t}^{\infty} e^{\lambda_{i 2}(t-s)} H_{i}\left(\phi_{1}, \phi_{2}, \ldots, \phi_{n}\right)(s) d s\right],
\end{aligned}
$$

where

$$
\lambda_{i 1}=\frac{c-\sqrt{c^{2}+4 D_{i} a_{i}}}{2 D_{i}}, \quad \lambda_{i 2}=\frac{c+\sqrt{c^{2}+4 D_{i} a_{i}}}{2 D_{i}} .
$$

It is easy to see that $F_{i}\left(\phi_{1}, \phi_{2}, \ldots, \phi_{n}\right)$ satisfy

$$
\begin{aligned}
& D_{i} F_{i}^{\prime \prime}\left(\phi_{1}, \phi_{2}, \ldots, \phi_{n}\right)-c F_{i}^{\prime}\left(\phi_{1}, \phi_{2}, \ldots, \phi_{n}\right) \\
& \quad-a_{i} F_{i}\left(\phi_{1}, \phi_{2}, \ldots, \phi_{n}\right)+H_{i}\left(\phi_{1}, \phi_{2}, \ldots, \phi_{n}\right)(t)=0 .
\end{aligned}
$$

Throughout this paper, we always assume that the following assumptions hold.

$\left(\mathrm{H}_{1}\right)$

$$
\begin{aligned}
& \sum_{j=1}^{n} w_{i j} f_{j}(0)+\sum_{j=1}^{n} h_{i j} g_{j}(0) \\
& =\sum_{j=1}^{n} w_{i j} f_{j}\left(k_{j}\right)+\sum_{j=1}^{n} h_{i j} g_{j}\left(k_{j}\right)=0, \quad i=1,2, \ldots, n .
\end{aligned}
$$

$\left(\mathrm{H}_{2}\right)$ For any $0 \leq \phi_{i}^{1}, \phi_{i}^{2} \leq k_{i}(i=1,2, \ldots, n)$, there exist some positive constants $L_{i}, K_{i}>0$ such that

$$
\begin{aligned}
& \left|f_{i}\left(\phi_{i}^{1}\right)-f_{i}\left(\phi_{i}^{2}\right)\right| \leq L_{i}\left|\phi_{i}^{1}-\phi_{i}^{2}\right|, \\
& \left|g_{i}\left(\phi_{i}^{1}\right)-g_{i}\left(\phi_{i}^{2}\right)\right| \leq K_{i}\left|\phi_{i}^{1}-\phi_{i}^{2}\right| .
\end{aligned}
$$


Let $u>0$ and equip $\Phi=\left(\phi_{1}, \phi_{2}, \ldots, \phi_{n}\right) \epsilon$ $C\left(R, R^{n}\right)$ with the exponential decay norm defined by $|\Phi|_{\mu}=$ $\sup _{t \in R} e^{-\mu|t|}|\Phi(t)|_{R^{n}}$. And define $B_{\mu}\left(R, R^{n}\right)=\left\{\Phi \in C\left(R, R^{n}\right)\right.$ : $\left.|\Phi|_{\mu}<\infty\right\}$; then it is easy to see that $\left(B_{\mu}\left(R, R^{n}\right),|\cdot|_{\mu}\right)$ is a Banach space.

We will study traveling wave solution to system (2) in the following profile set:

$$
\begin{aligned}
& \Gamma\left(\left(\underline{\phi}_{1}, \underline{\phi}_{2}, \ldots, \underline{\phi}_{n}\right),\left(\bar{\phi}_{1}, \bar{\phi}_{2}, \ldots, \bar{\phi}_{n}\right)\right) \\
& \quad=\left\{\begin{array}{l}
(1) \phi_{n} \text { is nondecreasing in } R ; \\
(2) \underline{\phi}_{i} \leq \phi_{i} \leq \bar{\phi}_{i}, \quad i=1,2, \ldots, n .
\end{array}\right.
\end{aligned}
$$

It is easy to check that $\Gamma\left(\left(\underline{\phi}_{1}, \underline{\phi}_{2}, \ldots, \underline{\phi}_{n}\right),\left(\bar{\phi}_{1}, \bar{\phi}_{2}, \ldots, \bar{\phi}_{n}\right)\right)$ is nonempty, convex, closed, and bounded.

Definition 1. Function $\bar{\Phi}=\left(\bar{\phi}_{1}, \bar{\phi}_{2}, \ldots, \bar{\phi}_{n}\right)$ is called upper solution of system (2) if $\bar{\Phi}$ is twice differentiable almost everywhere in $R$, and there hold

$$
\begin{aligned}
& D_{i} \bar{\phi}_{i}^{\prime \prime}(t)-c \bar{\phi}_{i}^{\prime}(t)-a_{i} \bar{\phi}_{i}(t) \\
&+H_{i}\left(\bar{\phi}_{1}, \bar{\phi}_{2}, \ldots, \bar{\phi}_{i}, \underline{\phi}_{i+1}, \bar{\phi}_{i+2}, \ldots, \bar{\phi}_{n}\right)(t) \\
& \leq 0, \quad \text { a.e. in } R, \\
& D_{n} \bar{\phi}_{n}^{\prime \prime}(t)-c \bar{\phi}_{n}^{\prime}(t)-a_{i} \bar{\phi}_{n}(t) \\
& \quad+H_{n}\left(\bar{\phi}_{1}, \bar{\phi}_{2}, \ldots, \bar{\phi}_{n}\right) \leq 0, \quad \text { a.e. in } R .
\end{aligned}
$$

Reversing the direction of above inequalities, we can get the lower solution.

In this paper, we assume that the upper-lower solutions of system $(2) \bar{\Phi}=\left(\bar{\phi}_{1}, \bar{\phi}_{2}, \ldots, \bar{\phi}_{n}\right)$ and $\underline{\Phi}=\left(\underline{\phi}_{1}, \underline{\phi}_{2}, \ldots, \underline{\phi}_{n}\right)$ satisfy

$$
\begin{aligned}
& \left(\mathrm{P}_{1}\right)(0,0, \ldots, 0) \leq\left(\underline{\phi}_{1}, \underline{\phi}_{2}, \ldots, \underline{\phi}_{n}\right) \leq\left(\bar{\phi}_{1}, \bar{\phi}_{2}, \ldots,\right. \\
& \left.\bar{\phi}_{n}\right) \leq\left(k_{1}, k_{2}, \ldots, k_{n}\right), t \in R \\
& \left(\mathrm{P}_{2}\right) \lim _{t \rightarrow-\infty}\left(\underline{\phi}_{1}, \underline{\phi}_{2}, \ldots, \underline{\phi}_{n}\right)=(0,0, \ldots, 0) \text {, and } \\
& \lim _{t \rightarrow \infty}\left(\bar{\phi}_{1}, \bar{\phi}_{2}, \ldots, \bar{\phi}_{n}\right)=\left(k_{1}, k_{2}, \ldots, k_{n}\right) .
\end{aligned}
$$

Lemma 2. Assume that (PQM) holds; then one has

(1) $F_{n}\left(\phi_{1}, \phi_{2}, \ldots, \phi_{n}\right)(t)$ is nondecreasing for $t \in R$.

(2) For $t \in R, i=1,2, \ldots, n-1$ and $0 \leq \underline{\phi}_{j} \leq \bar{\phi}_{j} \leq k_{j}(j=$ $1,2, \ldots, n)$, there has

$$
\begin{aligned}
& F_{i}\left(\underline{\phi}_{i}, \underline{\phi}_{2}, \ldots, \underline{\phi}_{i}, \bar{\phi}_{i+1}, \underline{\phi}_{i+2}, \ldots, \underline{\phi}_{n}\right)(t) \\
& \quad \leq F_{i}\left(\phi_{1}, \phi_{2}, \ldots, \phi_{n}\right)(t) \\
& \quad \leq F_{i}\left(\bar{\phi}_{i}, \bar{\phi}_{2}, \ldots, \bar{\phi}_{i}, \underline{\phi}_{i+1}, \bar{\phi}_{i+2}, \ldots, \bar{\phi}_{n}\right)(t) .
\end{aligned}
$$

Lemma 2 is easy to prove, so we omit it.
Lemma 3. Assume that $\left(H_{2}\right)$ holds; then $F_{i}\left(\phi_{1}, \phi_{2}, \ldots, \phi_{n}\right)(t)$ is continuous with respect to the norm $|\cdot|$ in $B_{\mu}\left(R, R^{n}\right)$.

Proof. For any fixed $\epsilon>0$, choose $\delta<\epsilon /\left(\sum_{j=1}^{n} w_{i j} L_{j}+\right.$ $\left.\sum_{j=1}^{n} h_{i j} K_{j}\right)$; a direct calculation shows that $\left|\phi_{j}^{1}-\phi_{j}^{2}\right|_{\mu}<\delta$, $\left|\phi_{j}^{1}\left(t-\tau_{j}(t)\right)-\phi_{j}^{2}\left(t-\tau_{j}(t)\right)\right|<\delta$; then there exists

$$
\begin{aligned}
\mid H_{i} & \left(\phi_{1}^{1}, \phi_{2}^{1}, \ldots, \phi_{n}^{1}\right)(t)-H_{i}\left(\phi_{1}^{2}, \phi_{2}^{2}, \ldots, \phi_{n}^{2}\right)(t) \mid e^{-\mu|t|} \\
= & \mid \sum_{j=1}^{n} w_{i j} f_{j}\left(\phi_{j}^{1}(t)\right)+\sum_{j=1}^{n} h_{i j} g_{j}\left(\phi_{j}\left(t-\tau_{j}(t)\right)\right) \\
& \quad-\sum_{j=1}^{n} w_{i j} f_{j}\left(\phi_{j}^{2}(t)\right)-\sum_{j=1}^{n} h_{i j} g_{j}\left(\phi_{j}^{2}\left(t-\tau_{j}(t)\right)\right) \mid e^{-\mu|t|} \\
\leq & \sum_{j=1}^{n} w_{i j}\left|f_{j}\left(\phi_{j}^{1}(t)\right)-f_{j}\left(\phi_{j}^{2}(t)\right)\right| e^{-\mu|t|} \\
& +\sum_{j=1}^{n} h_{i j} \mid g_{j}\left(\phi_{j}^{1}\left(t-\tau_{j}(t)\right)\right) \\
& \quad-g_{j}\left(\phi_{j}^{2}\left(t-\tau_{j}(t)\right)\right) \mid e^{-\mu|t|} \\
\leq & \sum_{j=1}^{n} w_{i j} L_{j} \delta+\sum_{j=1}^{n} h_{i j} K_{j} \delta \leq \epsilon .
\end{aligned}
$$

For $t>0$, we can see that

$$
\begin{aligned}
& \left|F_{i}\left(\phi_{1}^{1}, \phi_{2}^{1}, \ldots, \phi_{n}^{1}\right)-F_{i}\left(\phi_{1}^{2}, \phi_{2}^{2}, \ldots, \phi_{n}^{2}\right)\right| e^{-\mu|t|} \\
& \leq \frac{1}{D_{i}\left(\lambda_{i 2}-\lambda_{i 1}\right)} \\
& \quad \times\left[\int_{-\infty}^{t} e^{\lambda_{i 1}(t-s)}\right. \\
& \times \mid H_{i}\left(\phi_{1}^{1}, \phi_{2}^{1}, \ldots, \phi_{n}^{1}\right)(s) \\
& -H_{i}\left(\phi_{1}^{2}, \phi_{2}^{2}, \ldots, \phi_{n}^{2}\right)(s) \mid e^{-\mu|s|} e^{\mu|s|} d s \\
& +\int_{t}^{+\infty} e^{\lambda_{i 2}(t-s)} \\
& \quad \times \mid H_{i}\left(\phi_{1}^{1}, \phi_{2}^{1}, \ldots, \phi_{n}^{1}\right)(s) \\
& \quad-H_{i}\left(\phi_{1}^{2}, \phi_{2}^{2}, \ldots, \phi_{n}^{2}\right)(s) \mid \\
& \left.\times e^{-\mu|s|} e^{\mu|s|} d s\right] e^{-\mu t} \\
& \epsilon \quad\left[\int_{-\infty}^{t} e^{\lambda_{i 1}(t-s)} e^{\mu|s|} d s\right. \\
& \left.\quad+\int_{t}^{+\infty} e^{\lambda_{i 2}(t-s)} e^{\mu|s|} d s\right] e^{-\mu t}
\end{aligned}
$$




$$
\begin{aligned}
& =\frac{\epsilon}{D_{i}\left(\lambda_{i 2}-\lambda_{i 1}\right)}\left[\frac{2 \mu}{\lambda_{i 1}^{2}-\mu^{2}} e^{\left(\lambda_{i 1}-\mu\right) t}+\frac{\lambda_{i 2}-\lambda_{i 1}}{\left(\mu-\lambda_{i 1}\right)\left(\lambda_{i 2}-\mu\right)}\right] \\
& \leq \frac{\epsilon}{D_{i}\left(\lambda_{i 2}-\lambda_{i 1}\right)}\left[\frac{2 \mu}{\lambda_{i 1}^{2}-\mu^{2}}+\frac{\lambda_{i 2}-\lambda_{i 1}}{\left(\mu-\lambda_{i 1}\right)\left(\lambda_{i 2}-\mu\right)}\right] .
\end{aligned}
$$

For $t<0$, we have

$$
\begin{aligned}
& \left|F_{i}\left(\phi_{1}^{1}, \phi_{2}^{1}, \ldots, \phi_{n}^{1}\right)-F_{i}\left(\phi_{1}^{2}, \phi_{2}^{2}, \ldots, \phi_{n}^{2}\right)\right| e^{-\mu|t|} \\
& \leq \frac{1}{D_{i}\left(\lambda_{i 2}-\lambda_{i 1}\right)} \\
& \quad \times\left[\int_{-\infty}^{t} e^{\lambda_{i 1}(t-s)} \mid H_{i}\left(\phi_{1}^{1}, \phi_{2}^{1}, \ldots, \phi_{n}^{1}\right)(s)\right. \\
& -H_{i}\left(\phi_{1}^{2}, \phi_{2}^{2}, \ldots, \phi_{n}^{2}\right)(s) \mid e^{-\mu|s|} e^{\mu|s|} d s \\
& \quad+\int_{t}^{+\infty} e^{\lambda_{i 2}(t-s)} \mid H_{i}\left(\phi_{1}^{1}, \phi_{2}^{1}, \ldots, \phi_{n}^{1}\right)(s) \\
& \quad-H_{i}\left(\phi_{1}^{2}, \phi_{2}^{2}, \ldots, \phi_{n}^{2}\right)(s) \mid \\
& \leq \frac{\left.\epsilon e^{-\mu|s|} e^{\mu|s|} d s\right] e^{\mu t}}{D_{i}\left(\lambda_{i 2}-\lambda_{i 1}\right)}\left[\int_{-\infty}^{t} e^{\lambda_{i 1}(t-s)} e^{\mu|s|} d s\right. \\
& \left.\quad+\int_{t}^{+\infty} e^{\lambda_{i 2}(t-s)} e^{\mu|s|} d s\right] e^{\mu t} \\
& \leq \frac{\epsilon}{D_{i}\left(\lambda_{i 2}-\lambda_{i 1}\right)}\left[\frac{2 \mu}{\lambda_{i 2}^{2}-\mu^{2}}-\frac{\lambda_{i 2}-\lambda_{i 1}}{\left(\mu+\lambda_{i 1}\right)\left(\lambda_{i 2}+\mu\right)}\right] .
\end{aligned}
$$

So, $F_{i}: B_{\mu}\left(R, R^{n}\right) \rightarrow B_{\mu}\left(R, R^{n}\right)$ is continuous with respect to the norm $|\cdot|$ in $B_{\mu}\left(R, R^{n}\right)$.

Lemma 4. Assume that $\left(H_{1}\right)$ and $(P Q M)$ hold; then

$$
\begin{aligned}
& F\left(\Gamma\left(\left(\underline{\phi}_{1}, \underline{\phi}_{2}, \ldots, \underline{\phi}_{n}\right),\left(\bar{\phi}_{1}, \bar{\phi}_{2}, \ldots, \bar{\phi}_{n}\right)\right)\right) \\
& \quad \subset \Gamma\left(\left(\underline{\phi}_{1}, \underline{\phi}_{2}, \ldots, \underline{\phi}_{n}\right),\left(\bar{\phi}_{1}, \bar{\phi}_{2}, \ldots, \bar{\phi}_{n}\right)\right) .
\end{aligned}
$$

Proof. According to Lemma 2, for any $\left(\phi_{1}, \phi_{2}, \ldots, \phi_{n}\right)$ with $\left(\underline{\phi}_{1}, \underline{\phi}_{2}, \ldots, \underline{\phi}_{n}\right) \leq\left(\phi_{1}, \phi_{2}, \ldots, \phi_{n}\right) \leq\left(\bar{\phi}_{1}, \bar{\phi}_{2}, \ldots, \bar{\phi}_{n}\right)$, there exists

$$
\begin{aligned}
& F_{i}\left(\underline{\phi}_{i}, \underline{\phi}_{2}, \ldots, \underline{\phi}_{i}, \bar{\phi}_{i+1}, \underline{\phi}_{i+2}, \ldots, \underline{\phi}_{n}\right)(t) \\
& \quad \leq F_{i}\left(\phi_{1}, \phi_{2}, \ldots, \phi_{n}\right)(t) \\
& \quad \leq F_{i}\left(\bar{\phi}_{i}, \bar{\phi}_{2}, \ldots, \bar{\phi}_{i}, \underline{\phi}_{i+1}, \bar{\phi}_{i+2}, \ldots, \bar{\phi}_{n}\right)(t) ; \\
& F_{n}\left(\underline{\phi}_{1}, \underline{\phi}_{2}, \ldots, \underline{\phi}_{n}\right) \\
& \quad \leq F_{n}\left(\phi_{1}, \phi_{2}, \ldots, \phi_{n}\right) \leq F_{n}\left(\bar{\phi}_{1}, \bar{\phi}_{2}, \ldots, \bar{\phi}_{n}\right) .
\end{aligned}
$$

By the definition of upper-lower solutions, we have

$$
\begin{aligned}
& D_{i} \bar{\phi}_{i}^{\prime \prime}(t)-c \bar{\phi}_{i}^{\prime}(t)-a_{i} \bar{\phi}_{i}(t) \\
& \quad+H_{i}\left(\bar{\phi}_{i}, \bar{\phi}_{2}, \ldots, \bar{\phi}_{i}, \underline{\phi}_{i+1}, \bar{\phi}_{i+2}, \ldots, \bar{\phi}_{n}\right)(t) \leq 0 .
\end{aligned}
$$

Choosing $\left(\phi_{1}, \phi_{2}, \ldots, \phi_{n}\right)=\left(\bar{\phi}_{i}, \bar{\phi}_{2}, \ldots, \bar{\phi}_{i}, \underline{\phi}_{i+1}, \bar{\phi}_{i+2}, \ldots, \bar{\phi}_{n}\right)$ in (9) and denoting $\widetilde{\phi}_{i}=F_{i}\left(\bar{\phi}_{i}, \bar{\phi}_{2}, \ldots, \bar{\phi}_{i}, \underline{\phi}_{i+1}, \bar{\phi}_{i+2}, \ldots, \bar{\phi}_{n}\right)$, we get

$$
\begin{aligned}
& D_{i} \widetilde{\phi}_{i}^{\prime \prime}(t)-c \widetilde{\phi}_{i}^{\prime}(t)-a_{i} \widetilde{\phi}_{i}(t) \\
& \quad+H_{i}\left(\bar{\phi}_{1}, \bar{\phi}_{2}, \ldots, \bar{\phi}_{i}, \underline{\phi}_{i+1}, \bar{\phi}_{i+2}, \ldots, \bar{\phi}_{n}\right)(t)=0 .
\end{aligned}
$$

Setting $w_{i}(t)=\widetilde{\phi}_{i}-\bar{\phi}_{i}$ and combining (22) and (23), we have

$$
D_{i} w_{i}^{\prime \prime}-c w_{i}^{\prime}-a_{i} \geq 0
$$

Repeating the proof of Lemma 3.3 in Wu and Zou [17], we obtain $w_{i}(t) \leq 0$, which implies that $F_{i}\left(\phi_{1}, \phi_{2}, \ldots, \phi_{n}\right) \leq$ $\bar{\phi}_{i}(i=1,2, \ldots, n-1)$.

Next, choosing $\left(\phi_{1}, \phi_{2}, \ldots, \phi_{n}\right)=\left(\bar{\phi}_{i}, \bar{\phi}_{2}, \ldots, \bar{\phi}_{n}\right)$ in (9), we can get $F_{n}\left(\phi_{1}, \phi_{2}, \ldots, \phi_{n}\right) \leq \bar{\phi}_{n}$. Then, by a similar argument, we know that $F_{i}\left(\phi_{1}, \phi_{2}, \ldots, \phi_{n}\right) \geq \underline{\phi}_{i},(i=$ $1,2, \ldots, n-1)$, and $F_{n}\left(\phi_{1}, \phi_{2}, \ldots, \phi_{n}\right) \geq \underline{\phi}_{n}$; then

$$
\begin{aligned}
F( & \left.\Gamma\left(\left(\underline{\phi}_{1}, \underline{\phi}_{2}, \ldots, \underline{\phi}_{n}\right),\left(\bar{\phi}_{1}, \bar{\phi}_{2}, \ldots, \bar{\phi}_{n}\right)\right)\right) \\
& \subset \Gamma\left(\left(\underline{\phi}_{1}, \underline{\phi}_{2}, \ldots, \underline{\phi}_{n}\right),\left(\bar{\phi}_{1}, \bar{\phi}_{2}, \ldots, \bar{\phi}_{n}\right)\right) .
\end{aligned}
$$

This completes the proof.

Lemma 5. The above defined function $F$ is compact.

Proof. By the definition of $F$, we have

$$
\begin{aligned}
& F_{i}^{\prime}\left(\phi_{1}, \phi_{2}, \ldots, \phi_{n}\right)(t) \\
& =\frac{\lambda_{i 1} e^{\lambda_{i 1} t}}{D_{i}\left(\lambda_{i 2}-\lambda_{i 1}\right)} \int_{-\infty}^{t} e^{-\lambda_{i 1} s} H_{i}\left(\phi_{1}, \phi_{2}, \ldots, \phi_{n}\right)(s) d s \\
& \quad+\frac{\lambda_{i 2} e^{\lambda_{i 2} t}}{D_{i}\left(\lambda_{i 2}-\lambda_{i 1}\right)} \int_{t}^{\infty} e^{-\lambda_{i 2} s} H_{i}\left(\phi_{1}, \phi_{2}, \ldots, \phi_{n}\right)(s) d s .
\end{aligned}
$$


Thus,

$$
\begin{aligned}
& \left|F_{i}^{\prime}\left(\phi_{1}, \phi_{2}, \ldots, \phi_{n}\right)(t)\right|_{\mu} \\
& =\sup _{t \in R} \mid \frac{\lambda_{i 1} e^{\lambda_{i 1} t}}{D_{i}\left(\lambda_{i 2}-\lambda_{i 1}\right)} \\
& \times \int_{-\infty}^{t} e^{-\lambda_{i 1} s} H_{i}\left(\phi_{1}, \phi_{2}, \ldots, \phi_{n}\right)(s) d s \\
& +\frac{\lambda_{i 2} e^{\lambda_{i 2} t}}{D_{i}\left(\lambda_{i 2}-\lambda_{i 1}\right)} \\
& \times \int_{t}^{\infty} e^{-\lambda_{i 2} s} H_{i}\left(\phi_{1}, \phi_{2}, \ldots, \phi_{n}\right)(s) d s \mid e^{-\mu|t|} \\
& \leq \frac{-\lambda_{i 1}}{D_{i}\left(\lambda_{i 2}-\lambda_{i 1}\right)} \\
& \times \sup _{t \in R} e^{\lambda_{i 1} t-\mu|t|} \int_{-\infty}^{t} e^{-\lambda_{i 1} s}\left|H_{i}\left(\phi_{1}, \phi_{2}, \ldots, \phi_{n}\right)(s)\right| d s \\
& +\frac{\lambda_{i 2}}{D_{i}\left(\lambda_{i 2}-\lambda_{i 1}\right)} \\
& \times \sup _{t \in R} e^{\lambda_{i 2} t-\mu|t|} \int_{t}^{\infty} e^{-\lambda_{i 2} s}\left|H_{i}\left(\phi_{1}^{2}, \phi_{2}^{2}, \ldots, \phi_{n}^{2}\right)(s)\right| d s \\
& \leq \frac{-\lambda_{i 1}}{D_{i}\left(\lambda_{i 2}-\lambda_{i 1}\right)}\left|H_{i}\left(\phi_{1}^{2}, \phi_{2}^{2}, \ldots, \phi_{n}^{2}\right)(t)\right|_{\mu} \\
& \times \sup _{t \in R} e^{\lambda_{i 1} t-\mu|t|} \int_{-\infty}^{t} e^{-\lambda_{i 1} s} e^{\mu|s|} d s \\
& +\frac{\lambda_{i 2}}{D_{i}\left(\lambda_{i 2}-\lambda_{i 1}\right)}\left|H_{i}\left(\phi_{1}^{2}, \phi_{2}^{2}, \ldots, \phi_{n}^{2}\right)(t)\right|_{\mu} \\
& \times \sup _{t \in R} e^{\lambda_{i 2} t-\mu|t|} \int_{t}^{\infty} e^{-\lambda_{i 2} s} e^{\mu|s|} d s .
\end{aligned}
$$

We will complete the proof by two cases as follows:

(i) case $t>0$

$$
\begin{aligned}
& \left|F_{i}^{\prime}\left(\phi_{1}, \phi_{2}, \ldots, \phi_{n}\right)(t)\right|_{\mu} \\
& \leq \frac{-\lambda_{i 1}}{D_{i}\left(\lambda_{i 2}-\lambda_{i 1}\right)} \\
& \quad \times \sup _{t \in R} e^{\left(\lambda_{i 1}-\mu\right) t}\left[\int_{-\infty}^{0} e^{-\left(\lambda_{i 1}+\mu\right) s} d s+\int_{0}^{t} e^{\left(\mu-\lambda_{i 1}\right) s} d s\right] \\
& \quad \times\left|H_{i}\left(\phi_{1}, \phi_{2}, \ldots, \phi_{n}\right)(t)\right|_{\mu} \\
& \quad+\frac{\lambda_{i 2}}{D_{i}\left(\lambda_{i 2}-\lambda_{i 1}\right)} \\
& \quad \times \sup _{t \in R} e^{\left(\lambda_{i 2}-\mu\right) t} \int_{t}^{\infty} e^{\left(\mu-\lambda_{i 2}\right) s} d s\left|H_{i}\left(\phi_{1}, \phi_{2}, \ldots, \phi_{n}\right)(t)\right|_{\mu}
\end{aligned}
$$

$$
\begin{aligned}
\leq & \frac{\lambda_{i 1}}{D_{i}\left(\mu+\lambda_{i 1}\right)\left(\lambda_{i 2}-\lambda_{i 1}\right)}\left|H_{i}\left(\phi_{1}, \phi_{2}, \ldots, \phi_{n}\right)(t)\right|_{\mu} \\
& +\frac{\lambda_{i 2}}{D_{i}\left(\lambda_{i 2}-\mu\right)\left(\lambda_{i 2}-\lambda_{i 1}\right)}\left|H_{i}\left(\phi_{1}, \phi_{2}, \ldots, \phi_{n}\right)(t)\right|_{\mu} \\
= & \frac{1}{D_{i}\left(\lambda_{i 2}-\lambda_{i 1}\right)}\left[\frac{\lambda_{i 1}}{\mu+\lambda_{i 1}}+\frac{\lambda_{i 2}}{\lambda_{i 2}-\mu}\right] \\
& \times\left|H_{i}\left(\phi_{1}, \phi_{2}, \ldots, \phi_{n}\right)(t)\right|_{\mu} ;
\end{aligned}
$$

(ii) case $t<0$

$$
\begin{aligned}
&\left|F_{i}^{\prime}\left(\phi_{1}, \phi_{2}, \ldots, \phi_{n}\right)(t)\right|_{\mu} \\
& \leq \frac{-\lambda_{i 1}}{D_{i}\left(\lambda_{i 2}-\lambda_{i 1}\right)} \\
& \quad \times \sup _{t \in R} e^{\left(\lambda_{i 1}+\mu\right) t} \int_{-\infty}^{t} e^{-\left(\lambda_{i 1}+\mu\right) s} d s \\
& \quad \times\left|H_{i}\left(\phi_{1}, \phi_{2}, \ldots, \phi_{n}\right)(t)\right|_{\mu} \\
& \quad+\frac{\lambda_{i 2}}{D_{i}\left(\lambda_{i 2}-\lambda_{i 1}\right)} \\
& \quad \times \sup _{t \in R} e^{\left(\lambda_{i 2}+\mu\right) t}\left[\int_{t}^{0} e^{\left(-\lambda_{i 2}-\mu\right) s} d s+\int_{0}^{\infty} e^{\left(\mu-\lambda_{i 2}\right) s} d s\right] \\
& \quad \times\left|H_{i}\left(\phi_{1}, \phi_{2}, \ldots, \phi_{n}\right)(t)\right|_{\mu} \\
& \leq \frac{\lambda_{i 1}}{D_{i}\left(\mu+\lambda_{i 1}\right)\left(\lambda_{i 2}-\lambda_{i 1}\right)}\left|H_{i}\left(\phi_{1}, \phi_{2}, \ldots, \phi_{n}\right)(t)\right|_{\mu} \\
&+\frac{\lambda_{i 2}}{D_{i}\left(\lambda_{i 2}-\mu\right)\left(\lambda_{i 2}-\lambda_{i 1}\right)}\left|H_{i}\left(\phi_{1}, \phi_{2}, \ldots, \phi_{n}\right)(t)\right|_{\mu} \\
&= \frac{1}{D_{i}\left(\lambda_{i 2}-\lambda_{i 1}\right)}\left[\frac{\lambda_{i 1}}{\mu+\lambda_{i 1}}+\frac{\lambda_{i 2}}{\lambda_{i 2}-\mu}\right] \\
& \times\left|H_{i}\left(\phi_{1}, \phi_{2}, \ldots, \phi_{n}\right)(t)\right|_{\mu} \cdot
\end{aligned}
$$

According to the conditions in (PQM), we get that $\left|H_{i}\left(\phi_{1}, \phi_{2}, \ldots, \phi_{n}\right)(t)\right|_{\mu}$ is bounded by a positive number. Therefore, $\left|F_{i}^{\prime}\left(\phi_{1}, \phi_{2}, \ldots, \phi_{n}\right)(t)\right|_{\mu}$ is bounded. The above estimate for $F^{\prime}$ shows that $F\left(\Gamma\left(\left(\underline{\phi}_{1}, \underline{\phi}_{2}, \ldots, \underline{\phi}_{n}\right)\right.\right.$, $\left.\left.\left(\bar{\phi}_{1}, \bar{\phi}_{2}, \ldots, \bar{\phi}_{n}\right)\right)\right)$ is equicontinuous. It follows from Lemma 4 that $F\left(\Gamma\left(\left(\underline{\phi}_{1}, \underline{\phi}_{2}, \ldots, \underline{\phi}_{n}\right), \quad\left(\bar{\phi}_{1}, \bar{\phi}_{2}, \ldots, \bar{\phi}_{n}\right)\right)\right)$ is uniformly bounded.

Next, we define

$$
\begin{aligned}
& F^{m}\left(\phi_{1}, \phi_{2}, \ldots, \phi_{n}\right)(t) \\
& \quad= \begin{cases}(1) F\left(\phi_{1}, \phi_{2}, \ldots, \phi_{n}\right)(t), & t \in[-m, m] ; \\
(2) F\left(\phi_{1}, \phi_{2}, \ldots, \phi_{n}\right)(m), & t \in(m, \infty) ; \\
(3) F\left(\phi_{1}, \phi_{2}, \ldots, \phi_{n}\right)(-m), & t \in(-\infty,-m) .\end{cases}
\end{aligned}
$$


Then, for each $m \geq 1, F^{m}\left(\phi_{1}, \phi_{2}, \ldots, \phi_{n}\right)(t)$ is also equicontinuous and uniformly bounded. In the interval $[-m, m]$, it follows from Ascoli-Arzela theorem that $F^{m}$ is compact. On the other hand, $F^{m} \rightarrow F$ in $B_{\mu}\left(R, R^{n}\right)$ as $m \rightarrow \infty$, since

$$
\begin{aligned}
& \sup _{t \in R}\left|F^{m}\left(\phi_{1}, \phi_{2}, \ldots, \phi_{n}\right)(t)-F\left(\phi_{1}, \phi_{2}, \ldots, \phi_{n}\right)(t)\right| e^{-\mu|t|} \\
& =\sup _{t \in(-\infty,-m) \cup(m, \infty)} \mid F^{m}\left(\phi_{1}, \phi_{2}, \ldots, \phi_{n}\right)(t) \\
& \quad-F\left(\phi_{1}, \phi_{2}, \ldots, \phi_{n}\right)(t) \mid e^{-\mu|t|} \\
& \leq 2 K e^{-\mu m} \longrightarrow 0, \quad m \longrightarrow \infty .
\end{aligned}
$$

By Proposition 2.12 in [22], we have that $F$ : $\Gamma\left(\left(\underline{\phi}_{1}, \underline{\phi}_{2}, \ldots, \underline{\phi}_{n}\right),\left(\bar{\phi}_{1}, \bar{\phi}_{2}, \ldots, \bar{\phi}_{n}\right)\right) \rightarrow \Gamma\left(\left(\underline{\phi}_{1}, \underline{\phi}_{2}, \ldots, \underline{\phi}_{n}\right)\right.$, $\left.\left(\bar{\phi}_{1}, \bar{\phi}_{2}, \ldots, \bar{\phi}_{n}\right)\right)$ is compact. This completes the proof.

\section{Main Results}

Theorem 6. Assume that $\left(H_{1}\right),\left(H_{2}\right)$, and $(P Q M)$ hold. Moreover, suppose that there is a pair of upper-lower solutions $\bar{\Phi}=$ $\left(\bar{\phi}_{1}, \bar{\phi}_{2}, \ldots, \bar{\phi}_{n}\right)$ and $\underline{\Phi}=\left(\underline{\phi}_{1}, \underline{\phi}_{2}, \ldots, \underline{\phi}_{n}\right)$ for $(2)$ satisfying $\left(P_{1}\right)$ and $\left(P_{2}\right)$. Then, system (2) has a travelling wave solution.

Proof. Following Lemmas 2 to 5, we see that all the condition in Schauder's fixed point theorem hold. Then we know that there exists a fixed point $\left(\phi_{1}^{*}, \phi_{2}^{*}, \ldots, \phi_{n}^{*}\right)$ of $F$ in $\Gamma\left(\left(\underline{\phi}_{1}, \underline{\phi}_{2}, \ldots, \underline{\phi}_{n}\right),\left(\bar{\phi}_{1}, \bar{\phi}_{2}, \ldots, \bar{\phi}_{n}\right)\right)$. Now we need to verify the asymptotic boundary conditions. By $\left(\mathrm{P}_{2}\right)$ we notice the fact that

$$
\begin{aligned}
(0,0, \ldots, 0) & \leq\left(\underline{\phi}_{1}, \underline{\phi}_{2}, \ldots, \underline{\phi}_{n}\right) \leq\left(\phi_{1}^{*}, \phi_{2}^{*}, \ldots, \phi_{n}^{*}\right) \\
& \leq\left(\bar{\phi}_{1}, \bar{\phi}_{2}, \ldots, \bar{\phi}_{n}\right) \leq\left(k_{1}, k_{2}, \ldots, k_{n}\right)
\end{aligned}
$$

we get that

$$
\begin{aligned}
\lim _{t \rightarrow-\infty}\left(\phi_{1}^{*}, \phi_{2}^{*}, \ldots, \phi_{n}^{*}\right) & =(0,0, \ldots, 0), \\
\lim _{t \rightarrow \infty}\left(\phi_{1}^{*}, \phi_{2}^{*}, \ldots, \phi_{n}^{*}\right) & =\left(k_{1}, k_{2}, \ldots, k_{n}\right) .
\end{aligned}
$$

Therefore, the fixed point $\left(\phi_{1}^{*}, \phi_{2}^{*}, \ldots, \phi_{n}^{*}\right)$ satisfies the asymptotic boundary conditions. This completes the proof.

\section{Conclusion}

In this paper, we studied an n-species food chain model with spatial diffusion and time delays. By using Schauder's fixed point theorem and cross-iteration methods, we reduced the existence of the travelling wave solutions to the existence of a pair of upper-lower solutions. Finally, we proved that the system (2) has a travelling wave solution. However, in order to investigate the specific form of the travelling wave solution of (2), we still have a lot of work to do in the future.

\section{Conflict of Interests}

The authors declare that there is no conflict of interests regarding the publication of this paper.

\section{Acknowledgments}

This work was supported by the NNSF of China under Grant (no. 11271261), Natural Science Foundation of Shanghai (no. 13ZR1430100), Shanghai municipal education commission (n. 14ZZ12), and the Slovenian Research Agency, and a Marie Curie International Research Staff Exchange Scheme Fellowship within the 7th European Community Programme, FP7PEOPLE-2012-IRSES-316338.

\section{References}

[1] Y. Kuang, Delay Differential Equations with Applications in Population Dynamics, Mathematics in Science and Engineering, Academic Press, New York, NY, USA, 1993.

[2] R. Xu, M. A. J. Chaplain, and F. A. Davidson, "Periodic solution for athree-species Lotka-Volterra food-chain model with time delays," Mathematical and Computer Modelling, vol. 40, no. 7-8, pp. 823-837, 2004.

[3] X.-P. Yan and Y.-D. Chu, "Stability and bifurcation analysis for a delayed Lotka-Volterra predator-prey system," Journal of Computational and Applied Mathematics, vol. 196, no. 1, pp. 198210, 2006.

[4] C. V. Pao, "Global asymptotic stability of Lotka-Volterra 3species reaction-diffusion systems with time delays," Journal of Mathematical Analysis and Applications, vol. 281, no. 1, pp. 186204, 2003.

[5] C. V. Pao, "Global asymptotic stability of Lotka-Volterra competition systems with diffusion and time delays," Nonlinear Analysis: Real World Applications, vol. 5, no. 1, pp. 91-104, 2004.

[6] C. V. Pao, "The global attractor of a competitor-competitormutualist reaction-diffusion system with time delays," Nonlinear Analysis: Theory, Methods and Applications, vol. 67, no. 9, pp. 2623-2631, 2007.

[7] Y. Tang and L. Zhou, "Stability switch and Hopf bifurcation for a diffusive prey-predator system with delay," Journal of Mathematical Analysis and Applications, vol. 334, no. 2, pp. 1290-1307, 2007.

[8] K. W. Schaaf, "Asymptotic behavior and traveling wave solutions for parabolic functional-differential equations," Transactions of the American Mathematical Society, vol. 302, no. 2, pp. 587-615, 1987.

[9] R. A. Gardner, "Existence and stability of travelling wave solutions of competition models: a degree theoretic approach," Journal of Differential Equations, vol. 44, no. 3, pp. 343-364, 1982.

[10] J. Wu, Theory and Applications of Partial Functional-Differential Equations, Springer, New York, NY, USA, 1990.

[11] S. R. Dunbar, "Travelling wave solutions of diffuse LotkaVolterra equations," Journal of Mathematical Biology, vol. 17, no. 1, pp. 11-32, 1983.

[12] P. Bates and F. Chen, "Periodic traveling waves for a nonlocal integro-differential model," Electronic Journal of Differential Equations, vol. 1999, pp. 1-19, 1999.

[13] S. Ma and Y. Duan, "Asymptotic stability of traveling waves in a discrete convolution model for phase transitions," Journal of 
Mathematical Analysis and Applications, vol. 308, no. 1, pp. 240256, 2005.

[14] J. D. Murray, Mathematical Biology, Springer, Berlin, Germany, 1989.

[15] J. Li and Z. Liu, "Smooth and non-smooth traveling waves in a nonlinearly dispersive equation," Applied Mathematical Modelling, vol. 25, no. 1, pp. 41-56, 2000.

[16] K. Wang and W. Wang, "Propagation of HBV with spatial dependence," Mathematical Biosciences, vol. 210, no. 1, pp. 7895, 2007.

[17] J. Wu and X. Zou, "Traveling wave fronts of reaction-diffusion systems with delay," Journal of Dynamics and Differential Equations, vol. 13, no. 3, pp. 651-687, 2001.

[18] X. Zou and J. Wu, "Local existence and stability of periodic traveling waves of lattice functional-differential equations," The Canadian Applied Mathematics Quarterly, vol. 6, no. 4, pp. 397418, 1998.

[19] J. Huang and X. Zou, "Traveling wavefronts in diffusive and cooperative Lotka-Volterra system with delays," Journal of Mathematical Analysis and Applications, vol. 271, no. 2, pp. 455466, 2002.

[20] S. Ma, "Traveling wavefronts for delayed reaction-diffusion systems via a fixed point theorem," Journal of Differential Equations, vol. 171, no. 2, pp. 294-314, 2001.

[21] Q. Gan, R. Xu, X. Zhang, and P. Yang, "Travelling waves of a three-species Lotka-Volterra food-chain model with spatial diffusion and time delays," Nonlinear Analysis: Real World Applications, vol. 11, no. 4, pp. 2817-2832, 2010.

[22] E. Zeidler, Nonlinear Functional Analysis and its Applications: I. Fixed-Point Theorems, Springer, New York, NY, USA, 1986. 


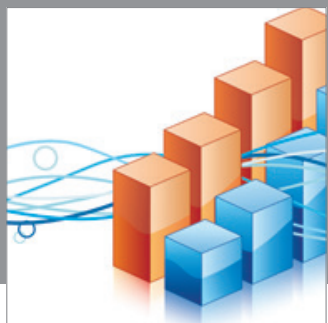

Advances in

Operations Research

mansans

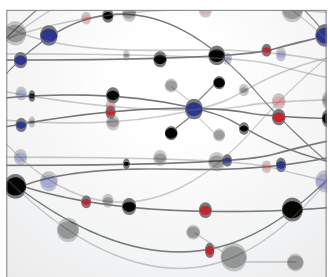

The Scientific World Journal

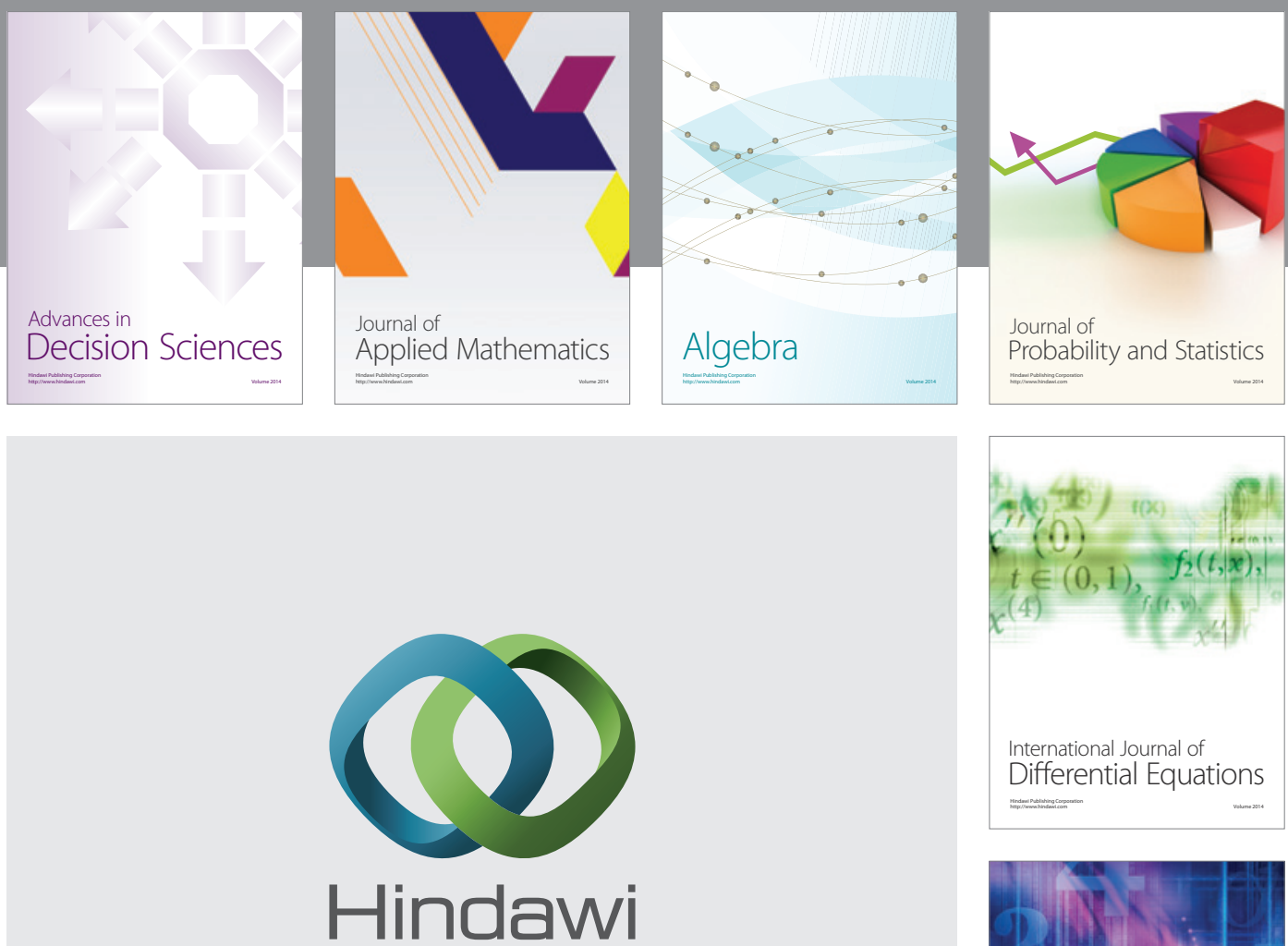

Submit your manuscripts at http://www.hindawi.com
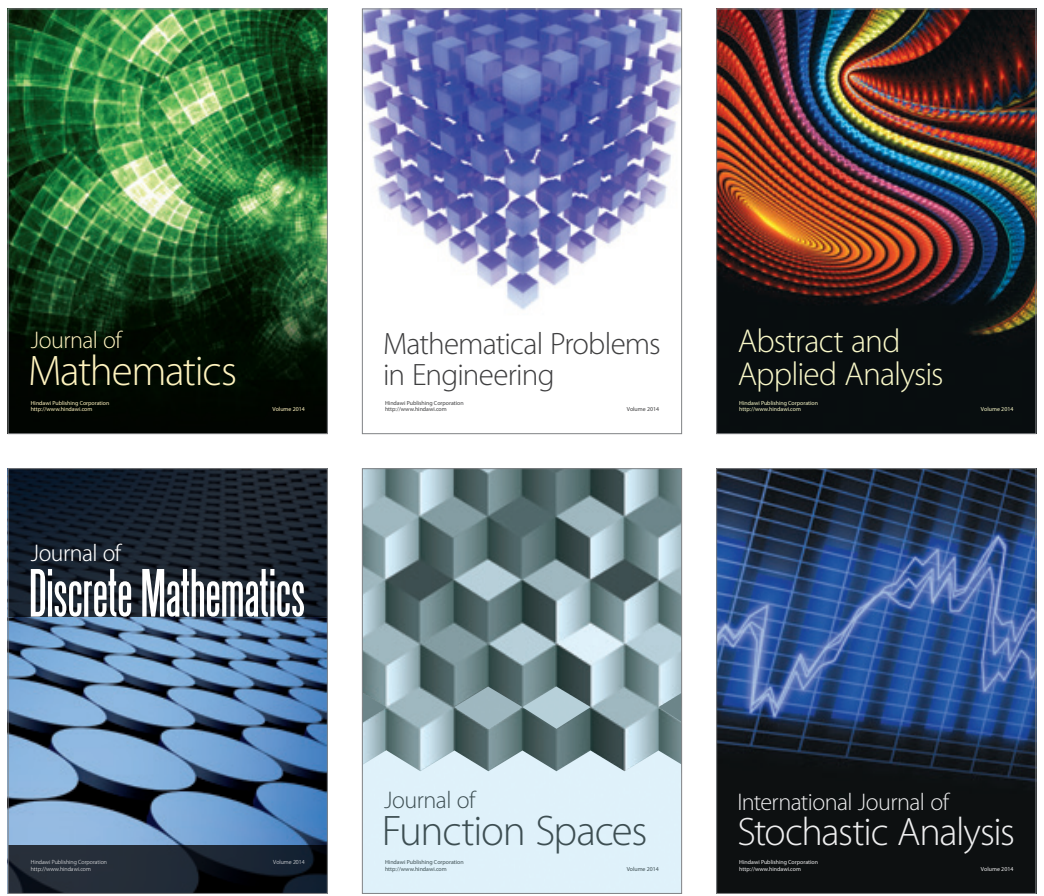

Journal of

Function Spaces

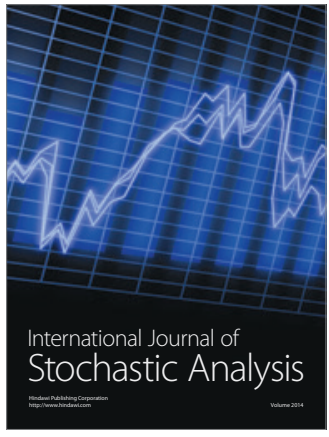

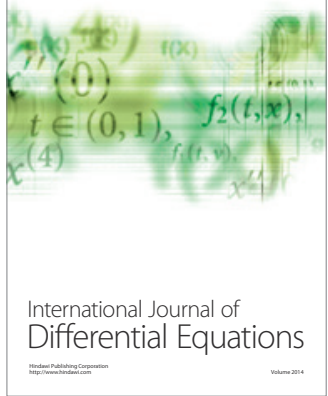
